\title{
Impétigo secondaire
}

\section{Secondary Impetigo}

\section{Tourdias $\cdot$ M. Daburon}

Reçu le 11 janvier 2016; accepté le 14 mars 2016

(C) SFMU et Lavoisier SAS 2016

Une patiente de 22 ans se présente aux urgences suite au développement depuis deux jours de lésions cutanées inquiétantes au niveau de son visage. L'interrogatoire retrouve, chez cette agricultrice saisonnière en bon état général, des piqûres de moustique prurigineuses quelques jours auparavant. L'inspection objective la présence de quatre lésions cutanées croûteuses jaunâtres, arrondies et de taille variable avec un halo inflammatoire périphérique (Fig. 1). Ce tableau permet d'évoquer une pyodermite superficielle aiguë par impétiginisation ou impétigo secondaire. L'évolution a été favorable, sans cicatrice, après un traitement local adapté. L'impétigo est une affection cutanée très fréquente d'origine bactérienne (Staphylococcus aureus et/ou Streptococcus pyogenes) qui atteint la couche cornée de l'épiderme et touche préférentiellement le jeune enfant [1]. L'impétigo primitif, forme rare, est une invasion bactérienne directe sur une peau préalablement saine. L'impétigo dit secondaire, forme habituelle, est une surinfection de lésions cutanées. Chez l'adulte, il complique classiquement une dermatose prurigineuse : piqûre d'insecte (comme le cas illustré ici) ou ectoparasitose dont la gale représente au niveau mondial une cause fréquente [2]. Le diagnostic repose exclusivement sur la clinique et, en l'absence de complication, il n'existe pas de signes généraux. Un prélèvement bactériologique n'est indiqué qu'en cas de doute diagnostique ou si l'identification du germe est nécessaire (immunosuppression, contexte d'épidémie et hospitalisation récente du patient ou d'un proche). La prise en charge thérapeutique de l'impétigo reste controversée [1]. Dans les formes localisées, le traitement local biquotidien pendant 8 à 10 jours est habituellement suffisant : lavage rigoureux à l'eau et au savon, associé à l'application d'un antiseptique et/ou d'une pommade antibiotique (mupirocine ou acide fusidique). L'application d'un émollient comme la vaseline pourrait favoriser l'élimination des croûtes. Un traitement antibiotique ayant une activité anti-

\footnotetext{
D. Tourdias $(\square) \cdot$ M. Daburon

Service d'accueil des urgences - SMUR - UHCD, centre hospitalier Sud Gironde, rue Paul Langevin, F-33210 Langon

e-mail : tourdiasdamien@yahoo.fr
}

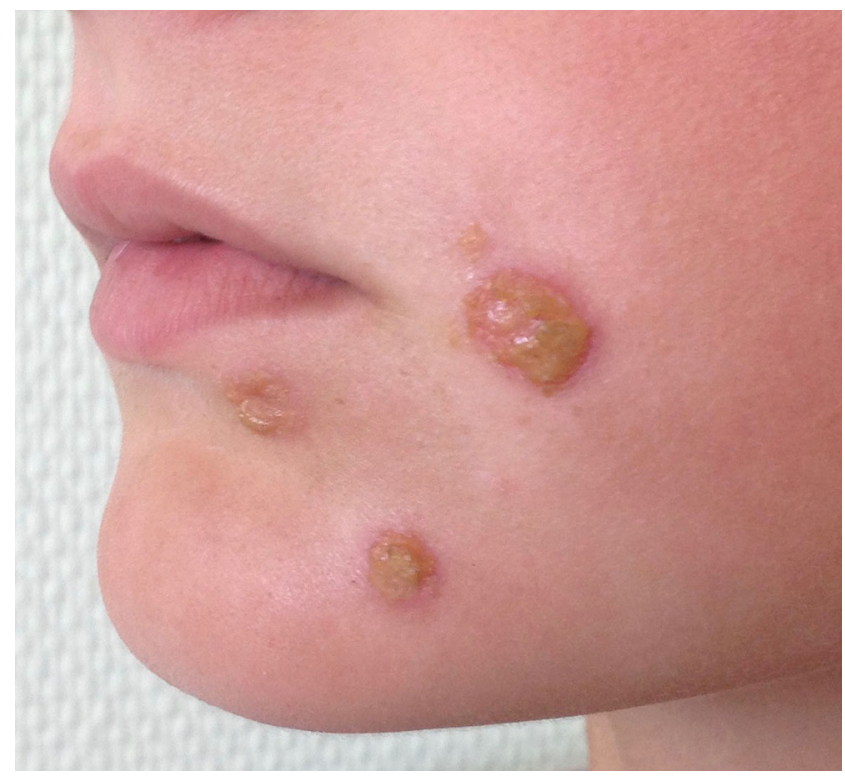

Fig 1 Impetigo secondaire non bulleux (forme la plus fréquente) chez un adulte. Notez l'aspect mélicérique (couleur miel) central caractéristique des croûtes et la localisation classique péribuccale

staphylococcique (pénicilline $\mathrm{M}$ ou pristinamycine) par voie systémique est nécessaire en cas de lésions étendues (en général plus de dix lésions ou plus de $2 \%$ de surface cutanée atteinte), de soins locaux incertains, de terrain débilité ou de signes généraux importants. Bien qu'exceptionnel, le principal risque est l'apparition d'une glomérulonéphrite aiguë poststreptococcique [1]. Ainsi, il est souhaitable de prévoir de façon systématique le dépistage d'une protéinurie à distance de l'épisode infectieux, en général à trois semaines, par le biais d'une simple bandelette urinaire.

\section{Références}

1. Hartman-Adams H, Banvard C, Juckett G (2014) Impetigo: diagnosis and treatment. Am Fam Physician 90:229-35

2. Romani L, Steer AC, Whitfeld MJ, Kaldor JM (2015) Prevalence of scabies and impetigo worldwide : a systematic review. Lancet Infect Dis 15:960-7 